\title{
Mental health and well-being during the COVID-19 pandemic: Iongitudinal analyses of adults in the UK COVID-19 Mental Health \& Wellbeing study
}

Rory C. O'Connor, Karen Wetherall, Seonaid Cleare, Heather McClelland, Ambrose J. Melson, Claire L. Niedzwiedz, Ronan E. O'Carroll, Daryl B. O'Connor, Steve Platt, Elizabeth Scowcroft, Billy Watson, Tiago Zortea, Eamonn Ferguson and Kathryn A. Robb

\section{Background}

The effects of coronavirus disease 2019 (COVID-19) on the population's mental health and well-being are likely to be profound and long lasting.

\section{Aims}

To investigate the trajectory of mental health and well-being during the first 6 weeks of lockdown in adults in the UK.

\section{Method}

A quota survey design and a sampling frame that permitted recruitment of a national sample was employed. Findings for waves 1 (31 March to 9 April 2020), 2 (10 April to 27 April 2020) and 3 (28 April to 11 May 2020) are reported here. A range of mental health factors was assessed: pre-existing mental health problems, suicide attempts and self-harm, suicidal ideation, depression, anxiety, defeat, entrapment, mental well-being and Ioneliness.

\section{Results}

A total of 3077 adults in the UK completed the survey at wave 1. Suicidal ideation increased over time. Symptoms of anxiety, and levels of defeat and entrapment decreased across waves whereas levels of depressive symptoms did not change significantly. Positive well-being also increased. Levels of loneliness did not change significantly over waves. Subgroup analyses showed that women, young people (18-29 years), those from more socially disadvantaged backgrounds and those with pre-existing mental health problems have worse mental health outcomes during the pandemic across most factors.

\section{Conclusions}

The mental health and well-being of the UK adult population appears to have been affected in the initial phase of the COVID-19 pandemic. The increasing rates of suicidal thoughts across waves, especially among young adults, are concerning.

\section{Keywords}

COVID-19; mental health; suicidal ideation; general population; depression.

\section{Copyright and usage}

(C) The Authors 2020. Published by Cambridge University Press on behalf of The Royal college of Psychiatrists. This is an Open Access article, distributed under the terms of the Creative commons Attribution licence (http://creativecommons.org/ licenses/by/4.0/), which permits unrestricted re-use, distribution, and reproduction in any medium, provided the original work is properly cited.

\section{Background}

The effects of coronavirus disease 2019 (COVID-19) on mental health and well-being are likely to be profound and long-lasting ${ }^{1,2}$ and will extend beyond those who have been directly affected by the virus. However, it is unclear who will be affected and to what extent such effects will generalise across all aspects of mental health.

Evidence from previous public health epidemics (for example the severe acute respiratory syndrome (SARS) virus) illustrated that the adverse effects are more common in some groups and that the detrimental effects are more pronounced among certain aspects of mental health than others. ${ }^{3-7}$ Increased risk of suicide was evident following SARS in older adults. ${ }^{4}$ Cross-sectional ${ }^{8-10}$ and longitudinal evidence (over 4 weeks) ${ }^{11}$ from China during the early stages of the outbreak of COVID-19 found high levels of mental health problems and distress in the general population. A study from Spain reported that distress during lockdown was associated with younger age and being female. ${ }^{12}$ Data from the University College London COVID-19 Social Study, which started post-pandemic, suggests self-harm and thoughts of suicide/selfharm were higher among women, Black, Asian and minority ethnic groups, people experiencing socioeconomic disadvantage and those with mental disorders. ${ }^{13}$ Repeated cross-sectional and longitudinal analysis of individual responses to the UK Household Longitudinal Study panel, including pre-pandemic data, have also demonstrated that mental health deteriorated in the early stages of the pandemic. ${ }^{14}$ All of these studies point to elevated rates of anxiety, depression, stress, suicide risk and post-traumatic stress in the initial stages of the pandemic.

On the 23 March 2020, a nationwide lockdown was announced by the UK government with the public instructed to stay at home, socially distance and self-isolate with strict guidance about movement outside of one's household. Public health measures are important to protect physical health, but it is essential that we gain a clearer understanding of the mental health and well-being of the UK population during the COVID-19 pandemic. ${ }^{15}$ Such understanding is vital to ensure that those affected receive the support that they require and to enable us to be better prepared for a potential second wave of the pandemic and for future outbreaks. Lockdown and the social and economic consequences of COVID-19 are likely to be associated with loneliness, social isolation and entrapment. ${ }^{1}$ To track their effects longitudinally, we assessed a wide range of mental health and well-being outcomes including: symptoms of depression and anxiety; well-being; defeat; entrapment; suicidal thoughts and behaviours; and loneliness.

\section{Aims}

The current study investigates the mental health and well-being of adults in the UK in the early weeks of the COVID-19 pandemic. Using a quota survey design and a sampling frame that permitted recruitment of a national sample, we report the mental health and 
well-being of adults in the UK at three time points across 6 weeks following the COVID-19 lockdown in the UK. We also investigated whether outcomes varied by sociodemographic characteristics and those with pre-existing mental health problems, given their established vulnerability. ${ }^{1,16,17}$

\section{Method}

\section{Study design, setting and participant recruitment}

Participant recruitment was conducted by Taylor McKenzie, a social research company. We recruited a non-probability sample of adults (aged 18 years or older) from across the UK to the UK COVID-19 Mental Health \& Wellbeing study (UK COVID-MH), with a longitudinal study design. We employed a quota sampling methodology, with quotas based on age (18-24 years: $12 \%$; $25-34$ : 17\%; 35-44: 18\%; 45-54: 18\%; 55-64: 15\%; $\geq 65$ : 20\%), gender (women: 51\%; men: 49\%), socioeconomic grouping (SEG; AB: 27\%; C1: 28\%; C2: 20\%; DE: 25\%, based on occupation, where A, $\mathrm{B}$ and $\mathrm{C} 1$ are higher and categories $\mathrm{C} 2, \mathrm{D}, \mathrm{E}$ are lower) and region of the UK (12 regions). The weighted and unweighted participant characteristics are presented in Table 1, with further details of gender identity and region reported in supplementary Tables 1 and 2. Weights are based on National Readership Survey and Office for National Statistics data for SEG and UK region, respectively and Census data for age and gender. ${ }^{18,19}$ Given the time sensitive nature of the study, a quota methodology was selected over probability sampling because it facilitated the recruitment of a well-stratified UK sample at the early phase of lockdown. Moreover, given the constraints of lockdown, online recruitment was the only feasible design.

Between 31 March and 9 April 2020, members of an existing online UK panel (Panelbase.net) were invited by email to take part in an online survey on health and well-being (wave 1). The panel has approximately 300000 registered adult members. In total, 7471 panel members were invited to take part, 3077 were included in the final sample (target sample was $n=3000$ ) and 4394 did not take part in the survey. The majority were screened out as a particular quota was full $(n=3527)$ and the remainder dropped out ( $n=867$; see Methods in the supplementary materials; available at https://doi.org/10.1192/bjp.2020.212). Respondents were asked demographic questions to determine whether they qualified for the study and if they did, they were re-directed to the survey.

After providing informed consent online, participants completed a wide range of psychological and social measures including questions about COVID-19. Only findings related to depression, anxiety, suicidal and self-harm history, defeat, entrapment, loneliness and well-being are reported here. Participants were informed that they would have the opportunity to participate in a minimum of six waves of the survey that would track the health and well-being of the UK during the current COVID-19 outbreak. All those who took part in wave 1 were invited to take part in the follow-up waves, and missing wave 2 did not exclude participation in wave 3. The follow-up surveys were scheduled to ensure a minimum of 1 week (wave 2) and 3 weeks (wave 3) between each participant completing a wave.

Three additional waves were scheduled between end of May and autumn 2020, with longer-term follow-ups also anticipated. This data collection schedule was decided to minimise fatigue effects and to maximise follow-up over time. In addition, we anticipated that after the initial shock of lockdown, changes in participants' well-being may be less marked over time thereby not requiring weekly data collection. Findings for waves 1 (31 March to 9 April 2020), 2 (10 April to 27 April 2020) and 3 (28 April to 11 May $2020)$ are reported here. At wave $2,89 \%(n=2742)$ completed the
Table 1 Demographic characteristics of the participants $(n=3077)$

\begin{tabular}{|c|c|c|}
\hline Characteristic & $\begin{array}{l}\text { Total, not } \\
\text { weighted: } \\
n(\%)\end{array}$ & $\begin{array}{c}\text { Total, weighted } \\
n(\%)\end{array}$ \\
\hline \multicolumn{3}{|l|}{ Gender at birth ${ }^{a}$} \\
\hline Men & $1381(44.9)$ & $1470(49.1)$ \\
\hline Women & $1692(55.1)$ & $1526(50.9)$ \\
\hline \multicolumn{3}{|l|}{ Ethnicity $^{\mathrm{b}}$} \\
\hline White & 2777 (90.5) & $2691(90.0)$ \\
\hline Asian & $162(5.3)$ & $169(5.7)$ \\
\hline Black & $68(2.2)$ & $72(2.4)$ \\
\hline Mixed & $52(1.7)$ & $48(1.6)$ \\
\hline Other & $10(0.3)$ & $10(0.3)$ \\
\hline \multicolumn{3}{|l|}{ Relationship status } \\
\hline Married/living with partner & $1834(59.6)$ & $1790(59.7)$ \\
\hline Single & $962(31.3)$ & $929(31.0)$ \\
\hline Separated/divorced/widowed & $248(8.1)$ & $247(8.2)$ \\
\hline Other/prefer not to say & $33(1.1)$ & $32(1.1)$ \\
\hline \multicolumn{3}{|l|}{ Sexuality } \\
\hline Heterosexual & $2830(92.0)$ & $2762(92.1)$ \\
\hline Gay or bisexual & $220(7.1)$ & $212(7.1)$ \\
\hline Other/prefer not to say & $27(0.9)$ & $26(0.9)$ \\
\hline \multicolumn{3}{|l|}{ Employment status } \\
\hline Employed & $1838(59.7)$ & $1806(60.2)$ \\
\hline Unemployed & $358(11.6)$ & $342(11.4)$ \\
\hline Other (retired, education, homemaker) & $881(28.6)$ & $852(28.4)$ \\
\hline \multicolumn{3}{|l|}{ Socioeconomic grouping ${ }^{c}$} \\
\hline High & $1758(57.1)$ & $1651(55.0)$ \\
\hline LOW & 1319 (42.9) & $1349(45.0)$ \\
\hline \multicolumn{3}{|l|}{ Tenure } \\
\hline Own (including with mortgage) & $1835(59.6)$ & $1792(59.7)$ \\
\hline Private rent & $694(22.6)$ & $682(22.7)$ \\
\hline Council rent & $463(15.0)$ & $446(14.9)$ \\
\hline Other & $85(2.8)$ & $81(2.7)$ \\
\hline Pre-existing mental health condition & $836(27.2)$ & $780(26.0)$ \\
\hline \multicolumn{3}{|c|}{$\begin{array}{l}\text { a } n=3073 . \\
{ }^{\mathrm{D}} n=3069 . \\
{ }^{\mathrm{C}} \text { Categories A,B,C1, high socioeconomic group; categories C2, D, E low socioeconomic } \\
\text { group. }\end{array}$} \\
\hline
\end{tabular}

survey and at wave 3 the survey was completed by $85 \%(n=2604$; see Fig. 1 for flow chart of participants across the waves).

The authors assert that all procedures contributing to this work comply with the ethical standards of the relevant national and institutional committees on human experimentation and with the Helsinki Declaration of 1975, as revised in 2008. All procedures involving human patients were approved by the University of Glasgow's Medical, Veterinary \& Life Sciences Ethics Committee (approval number: 200190146). The study was pre-registered at aspredicted.org (\#41910). Participants received $£ 1.50$ for the completion of the surveys and were entered into prize draws. A list of mental health support organisations was made available to participants online.

\section{Measures}

Suicidal history was assessed via two items adapted from the Adult Psychiatric Morbidity Survey, ${ }^{20}$ 'Have you ever made an attempt to take your life, e.g. by taking an overdose of tablets or in some other way?' (yes/no) (suicide attempt history) and 'Have you ever deliberately harmed yourself in any way but not with the intention of killing yourself?' (yes/no) (self-harm history). If respondents answered yes to the suicide attempt or the self-harm history questions, they were asked 'when was the last time you deliberately harmed yourself?' (past week, past month, past 6 months, more than 6 months, more than 12 months). We report self-harm and suicide attempts in the past week. Suicidal ideation in the past week was assessed by the question 'How often have you thought about taking your life in the last week? ('one day', 'several days', 


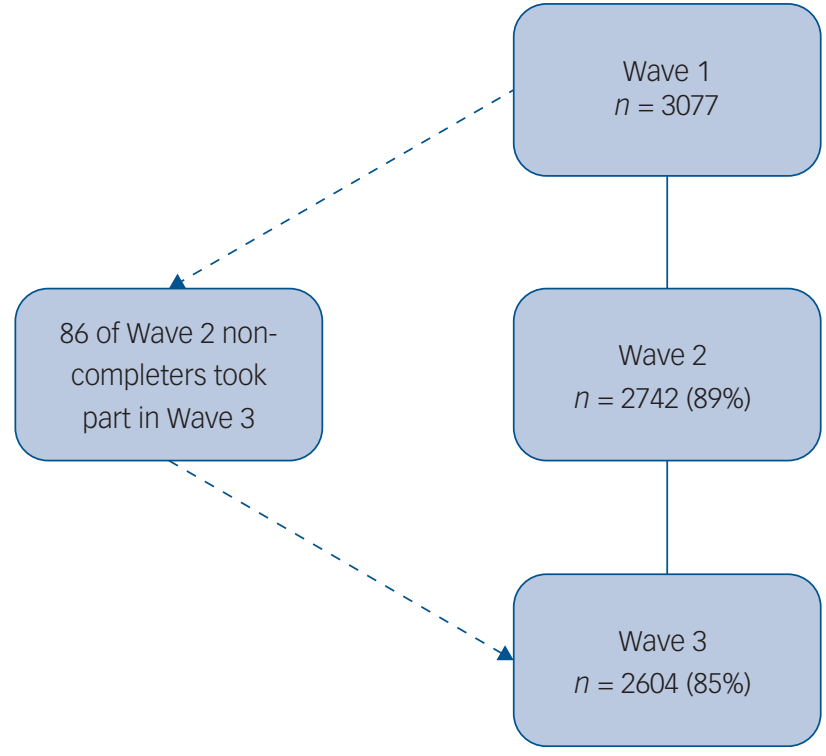

Fig. 1 Flow of participants across waves.

'more than half the days', 'nearly everyday', 'never', 'I would rather not answer')'. 'One day' to 'nearly everyday' was coded as yes, 'never' as no.

Depressive symptoms were assessed via the nine-item Patient Health Questionnaire (PHQ-9 ${ }^{21}$ ). The 7-item Generalized Anxiety Disorder (GAD-7) ${ }^{22}$ tool was used to assess symptoms of generalised anxiety disorder. Both measures ask how often symptoms are bothering the respondents in the past 2 weeks. Scores of $\geq 10$ on both measures are thought to indicate moderate levels of depression and anxiety and are used as cut-off points here. ${ }^{22,23}$

Feelings of defeat (perceived failed struggle and loss of rank) were assessed using four items from Griffiths' short-form scale. ${ }^{24}$ The Entrapment Scale Short-form ${ }^{25}$ was used to explore perceptions of entrapment (feeling trapped by thoughts and feelings or situation). Mental well-being was assessed via the 7-item Short Warwick Edinburgh Mental Well-Being Scale (SWEMWBS). ${ }^{26}$ Loneliness was assessed using the UCLA 3-item scale. ${ }^{27}$ The National Readership Survey social grade ${ }^{18}$ was used as an indicator of socioeconomic group (SEG): high $(\mathrm{A}+\mathrm{B}+\mathrm{C} 1)$ versus low $(\mathrm{C} 2+\mathrm{D}+\mathrm{E})$.

To assess pre-existing mental health conditions, participants were first asked if they had any long-standing physical or mental impairment, illness or disability. Participants were then asked to select their mental or physical impairment from a list of options, which included mental health conditions, neuro-divergent disorders and alcohol and drug problems, and these responses were used to create a dichotomous variable for presence or absence of a pre-existing mental health condition (see Table 2 of the Methods in the supplementary materials).

\section{Statistical analysis}

All analyses were conducted using Stata MP 16. Our analyses were conducted using an imputed data-set of the 3077 participants who completed the survey at wave 1 as there were significant differences in the mental health of those who did and did not complete all waves of the survey. We used multiple imputation (MI) to generate 50 data-sets for each outcome variable. MI generalised estimating models (MI-GEE) were then constructed to test the changes in the variables across waves for the whole sample. This approach is suitable for longitudinal data. ${ }^{28,29}$ As a sensitivity check, we ran all analyses with and without MI and found a similar pattern for both analyses. The GEE results presented here are those using MI. GEE models use a multilevel approach and produce odds ratios (ORs)

For the binary outcome variables (suicidal ideation, PHQ-9 and GAD-7 cut-off scores) binomial logit modelling was used, and for the continuous outcome variables (defeat, entrapment, loneliness, and positive well-being) linear Gaussian identity modelling was used. Region (South England, English Midlands, North England, Scotland, Wales and Northern Ireland) was controlled for in all analyses. We modelled the temporal covariation using an unstructured correlation matrix, as the pattern of associations was neither fully exchangeable nor had an first-order autoregressive structure. Further GEE models were conducted to test for subgroup differences in the outcome variables: as a function of age (18-29, $30-59, \geq 60$ years), gender (men, women), ethnicity (categorised into White and minority ethnic group because of small numbers), SEG (higher, lower) and the presence of a pre-existing mental health problem (no, yes).

Additionally, interactions between each of these subgroups and changes in each outcome over the waves was also tested, with only significant interactions reported in the results. As before, the binary variables were analysed using binomial logit GEE models and the continuous variables were explored using linear Gaussian identity GEE models.

Given the large number of analyses, a false discovery rate (FDR) was applied to all the between, within and interaction $P$-values from all analyses. FDR is a method of understanding the rate of type I errors in null hypothesis testing when conducting multiple comparisons. FDR-controlling procedures are designed to control the expected proportion of 'discoveries' that are false. ${ }^{30}$ There were few missing data at wave 1; a small number of participants indicated that they 'would rather not say' for the suicidal ideation $(n=93,3 \%$ at wave $1 ; n=91,3.3 \%$ at wave $2 ; n=71,2.7 \%$ at wave 3 ), suicide attempts $(n=71,2.3 \%$ at wave $1 ; n=36,1.3 \%$ at wave $2 ; n=32$; $1.2 \%$ at wave 3$)$ or self-harm $(n=64,2.1 \%$ at wave $1 ; n=39,1.4 \%$ at wave $2 ; n=33,1.3 \%$ at wave 3 ) questions; these were imputed via MI. As the rates of self-harm and suicide attempts in the past week were found to be low, no inferential statistics were applied to these data.

\section{Results}

\section{Participant characteristics}

A total of 3077 adults completed the survey at wave 1 (see Table 1, Fig. 1). In the unweighted data at wave $1,55.1 \%$ of the participants were women and $90.5 \%$ were White. Regarding age, $27.5 \%$ were aged $18-29$ years, $53.2 \%$ aged $30-59$ and $19.3 \%$ aged $\geq 60$ years. In total, $59.6 \%$ were married/living with partner and $92.0 \%$ selfidentified as heterosexual. Over half (57.1\%) reported occupations that were classified as higher SEG and 59.6\% reported owning their own home (including with mortgage). Just over a quarter $(27.2 \%)$ of the participants reported having a pre-existing mental health problem at wave 1 (see Table S6 in the supplementary materials).

\section{Mental health outcomes across waves for all adults}

Rates of suicidal ideation increased over time (see Table 2), with respondents at wave $2(9.2 \% ; \mathrm{OR}=1.17,95 \%$ CI $1.01-1.34$, $P=0.031)$ and wave $3(9.8 \% ; \mathrm{OR}=1.24,95 \% \mathrm{CI} 1.07-1.44$, $P=0.005)$ reporting higher levels than at wave $1(8.2 \%)$. The difference between waves 2 and 3 was not statistically significant. 


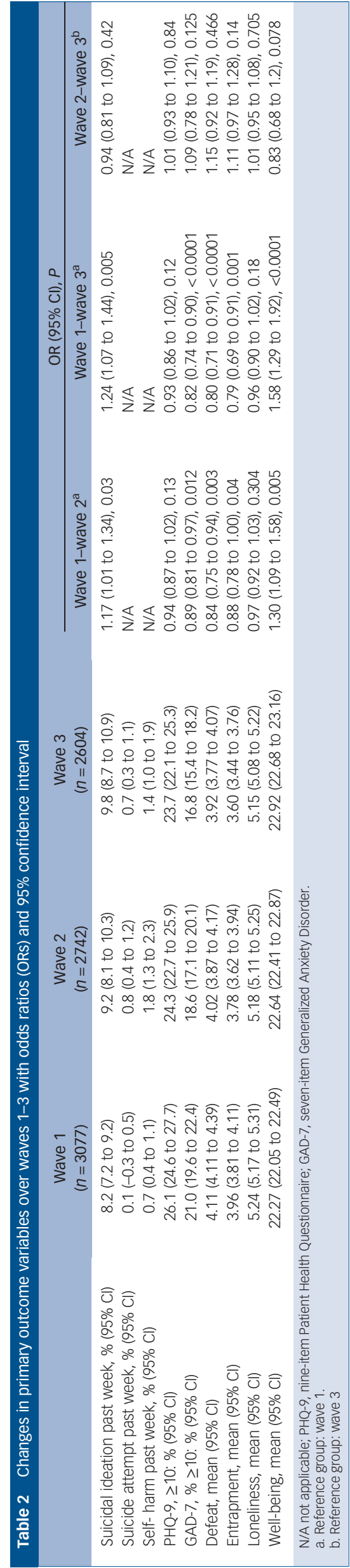

The rates of suicide attempt $(0.1 \%$ at wave $1,0.7 \%$ at wave 3$)$ and self-harm $(0.7 \%$ at wave 1 and $1.4 \%$ at wave 3$)$ in the past week were low.

A total of $21 \%$ of the participants was above the cut-off point for moderate or severe levels of symptoms of anxiety at wave 1. However, these symptoms decreased across waves, with wave 2 (18.6\%; OR $=0.89,95 \%$ CI $0.81-0.97, P=0.012)$ and wave 3 (16.8\%; OR $=0.82,95 \%$ CI $0.74-0.90, P<0.0001)$ being lower than wave $1(21 \%)$; the differences between wave 2 and wave 3 was not significant. More than a quarter $(26.1 \%)$ scored above the cut-off for moderate or severe levels of depression; there was no significant change across the waves.

Feelings of defeat decreased from wave 1 (mean 4.11) to wave 2 (mean 4.02; $\mathrm{OR}=0.84,95 \% \mathrm{CI} 0.75-0.95, P=0.003$ ) and from wave 1 to wave 3 (mean 3.92; $\mathrm{OR}=0.80,95 \%$ CI $0.71-0.91, P<0.0001$; Table 2). There was no difference between waves 2 and 3 . Entrapment also decreased over time, from wave 1 (mean 3.96) to wave 2 (mean 3.78; $\mathrm{OR}=0.88,95 \%$ CI $0.79-1.00, P=0.04$ ) and from wave 1 to wave 3 (mean 3.60; OR $=0.79,95 \%$ CI $0.69-0.91$, $P=0.001$ ) but not between waves 2 and 3 . Positive well-being increased from wave 1 (mean 22.27) to wave 2 (mean 22.64; $\mathrm{OR}=1.30,95 \%$ CI 1.09-1.58, $P=0.005$ ) and from wave 1 to wave 3 (mean 22.92; $\mathrm{OR}=1.58,95 \%$ CI 1.29-1.92, $P<0.0001$ ), but not from wave 2 to wave 3 . Levels of loneliness did not significantly change over waves.

\section{Mental health outcomes across wave by sociodemographic characteristics and pre-existing mental health condition}

Percentages and means from wave 1 have been used to illustrate differences between subgroups, although data from all waves were used in this analysis and are included in the supplementary material (see supplementary Tables 3-8).

\section{Suicidal ideation}

Men and women reported similar levels of suicidal ideation (see supplementary Table 3). Compared with younger adults (18-29 year olds; wave $112.5 \%)$ those aged $30-59$ years $(8.4 \%$; OR $=0.65$, $95 \%$ CI $0.49-0.85, P=0.002)$ and $\geq 60$ years $(1.9 \%$; $\mathrm{OR}=0.14$, $95 \%$ CI $0.08-0.27, P<0.0001)$ reported lower levels of suicidal ideation, and those aged 30-59 were more likely to report suicidal ideation than $\geq 60$-year-olds $(\mathrm{OR}=4.51,95 \%$ CI 2.43 $8.39, P<0.0001)$. There were no clear differences when comparing ethnic minorities to the White ethnic group (see supplementary Table 4). Those from the lower SEG were more likely to experience suicidal ideation $(10.3 \%$; OR $=1.63,95 \%$ CI $1.24-2.10, P<0.0001)$ compared with those in the higher SEG (6.6\%; see supplementary Table 5). Those with a pre-existing mental health condition were more likely to experience suicidal ideation $(19.3 \%$; OR $=5.56,95 \%$ CI 4.23-7.31, $P<0.0001)$ compared with those without $(4.1 \%$; see supplementary Table 6).

\section{Depressive symptoms}

Men reported lower levels of depressive symptoms (17.6\%) than women (33\%; $\mathrm{OR}=0.44,95 \%$ CI $0.37-0.52, \quad P<0.0001$; see supplementary Table 3$)$. Those who were aged $30-59$ years $(26 \%$; OR $=0.55,95 \%$ CI $0.46-0.66, P<0.0001)$ and aged $\geq 60$ years (8.2\%; OR $=0.14,95 \%$ CI $0.10-0.20, P<0.0001)$ reported lower levels of depressive symptoms than younger adults (38.8\%; $18-29$ years), and those aged 30-59 years reported higher rates of depressive symptoms than those $\geq 60 \quad(\mathrm{OR}=3.85,95 \%$ CI 2.82-5.27, $P<0.0001)$. There were no significant differences by ethnicity. The respondents in the lower SEG were more likely to report higher levels of depressive symptoms $(30.4 \%$; OR $=1.47$, 
95\% CI 1.25-1.73, $P<0.0001)$ than those in the higher SEG group (22.9\%; see supplementary Table 5).

People with a pre-existing mental health condition were significantly more likely to report higher levels of depressive symptoms (54.2\%; OR =6.50, 95\% CI 5.45-7.77, $P<0.0001$ ) compared with those without (15.3\%). The interaction between pre-existing mental health condition and wave was statistically significant (OR $=0.87,95 \%$ CI $0.79-0.96, P=0.007$ ), with those who had a pre-existing mental health condition reporting reductions in depressive symptoms over time at both wave 2 (reduction 5.6\%; $\mathrm{OR}=0.80,95 \%$ CI $0.67-0.96, P=0.017$ ) and wave 3 (reduction $7.5 \%$; OR $=0.76,95 \%$ CI 0.63-0.94, $P=0.009$ ), but not from wave 2 to wave 3 (reduction 1.9\%). Those with no pre-existing mental health conditions did not change over time.

\section{Anxiety symptoms}

Across the three waves, those aged $30-59$ years $(21.5 \%$; OR $=0.63$, $95 \%$ CI $0.52-0.76, P<0.0001)$ and those aged $\geq 60(6.4 \%$; OR $=0.16$, $95 \%$ CI $0.11-0.23, P<0.0001)$ were less likely to score above the cut-off for anxiety symptoms compared with those aged 18-29 years $(30.1 \%)$, and those age $30-59$ years were more likely to be above the cut-off for anxiety symptoms than those $\geq 60$ (OR $=3.95$, 95\% CI 2.79-5.61, $P<0.0001$; supplementary Table 3). Men were also less likely to meet the cut-off threshold (13\%) compared with women $(27.5 \%$; OR $=0.40,95 \%$ CI $0.33-0.48, P<0.0001)$. Levels of anxiety did not vary by ethnicity. Those who were of a lower SEG were more likely to score above the cut-off for anxiety symptoms (24.9\%; OR $=1.49,95 \%$ CI 1.25-1.78, $P<0.0001)$ compared with those in the higher SEG (18\%). Participants with a pre-existing mental health condition were more likely to score above the cut-off (44.6\%; OR $=5.97,95 \%$ CI 4.95-7.19, $P<0.0001)$ than those with no mental health condition (11.9\%; supplementary Table 6).

\section{Defeat}

Compared with those aged 18-29 years (mean 5.27), participants aged 30-59 years (mean 4.38; OR $=0.40,95 \%$ CI $0.29-0.55$, $P<0.0001$ ) and those aged $\geq 60$ years (mean 2.45 ; OR $=0.06,95 \%$ CI $0.04-0.09, P<0.0001)$ reported lower levels of defeat, and 30-59 year olds scored higher than those aged $\geq 60$ years $(\mathrm{OR}=6.77, \quad 95 \% \quad$ CI $4.72-9.71, \quad P<0.0001$; supplementary Table 7). Men reported significantly lower levels of defeat than females $(\mathrm{OR}=0.22,95 \%$ CI $0.17-0.29, P<0.0001)$. No differences were found by ethnicity on levels of defeat. Participants from a lower SEG reported higher levels of defeat (mean 4.81; OR $=2.69$, 95\% CI 2.03-3.56, $P<0.0001)$ compared with those of a higher SEG (mean 3.83; see supplementary Table 8). Participants who reported a pre-existing mental health condition reported higher levels of defeat (mean 7.06; OR $=48.47,95 \%$ CI 36.56-64.27, $P<0.0001)$ compared with those without a mental health condition (mean 3.17; see supplementary Table 8 ).

\section{Entrapment}

Men reported lower levels of entrapment (mean 3.14) than women (mean 4.62; OR $=0.23,95 \%$ CI $0.17-0.31, P<0.0001$ ). Levels of entrapment differed significantly by age group, with those aged $30-59$ years (mean 4.1 ; OR $=0.37,95 \%$ CI $0.26-0.53, P<0.0001$ ) and those aged $\geq 60$ years (mean 1.93; OR $=0.05,95 \% \mathrm{CI}$ $0.03-0.07, P<0.0001)$ reporting lower levels of entrapment than those aged 18-29 years (mean 5.07), and those aged $30-59$ years were higher than those aged $\geq 60(\mathrm{OR}=8.30,95 \%$ CI 5.58-12.34, $P<0.0001)$. No significant differences by ethnicity were found. Those from a lower SEG reported significantly higher levels of entrapment (mean 4.47; OR $=2.48,95 \%$ CI 1.82-3.38, $P<0.0001$ ) than those in the higher SEG group (mean 3.57). Participants with a pre-existing mental health condition reported higher levels of entrapment (mean 7.0; OR $=66.78,95 \%$ CI 48.99-91.04, $P<0.0001$ ) than those without (mean 2.79). The interaction between mental health condition and entrapment over the waves was significant $(\mathrm{OR}=0.84,95 \% \mathrm{CI} 0.72-0.98, P=0.02)$, with those who had a mental health condition experiencing a more pronounced reduction in the average entrapment score from wave 1 to wave 3 (reduction 0.63 ; OR $=0.70,95 \%$ CI $0.52-0.95, P=0.02$ ) compared with those with no mental health condition (reduction 0.2 ).

\section{Loneliness}

Men reported significantly lower levels of loneliness (mean 4.89) than women (mean 5.52; OR $=0.54,95 \%$ CI 0.47-0.62, $P<0.0001$ ). There were significant differences between the age groups on levels of loneliness, with those aged 30-59 years (mean 5.28; OR $=0.54,95 \%$ CI $0.46-0.63, P<0.0001$ ) and those aged $\geq 60$ (mean 4.31; OR $=0.21,95 \%$ CI $0.17-0.26, P<0.0001$ ) reporting lower levels of loneliness than those aged 18-29 years (mean 5.87), and those aged 30-59 years reporting higher loneliness than the $\geq 60$ group ( $\mathrm{OR}=2.54,95 \% \mathrm{CI} 2.13-3.02, P<0.0001)$. Additionally, the interaction between age and loneliness over the waves was significant $(\mathrm{OR}=1.06,95 \% \mathrm{CI} 1.01-1.11, P=0.016)$, as levels of loneliness reduced significantly for those aged 18-29 years from wave 1 to wave 2 (reduction 0.17 ; OR $=0.78,95 \%$ CI $0.65-0.92, P=0.004$ ) and wave 1 to wave 3 (reduction 0.21 ; OR $=0.81,95 \%$ CI $0.67-0.97$, $P=0.004)$ compared with those aged $\geq 60$ years, whose self-reported loneliness increased (wave 1 to wave 2 increase of 0.11 ).

Levels of loneliness did not differ by ethnic group. Those from the lower SEG (mean 5.39; OR =1.31, 95\% CI 1.14-1.50, $P<0.0001$ ) reported significantly higher levels of loneliness compared with those from the higher SEG (mean 5.12). Participants with a pre-existing mental health condition reported significantly higher levels of loneliness than (mean 6.28; OR $=4.19,95 \%$ CI 3.63-4.85, $P<0.0001$ ) those without (mean 4.24; see supplementary Table 8 ). There was evidence of a wave $\times$ mental health problem interaction $(\mathrm{OR}=0.89$, $95 \%$ CI $0.83-0.96, P=0.002$ ), with a significant decrease in loneliness in those who had a pre-existing mental health problem from wave 1 to wave 3 (reduction 0.26 ; $\mathrm{OR}=0.80,95 \% \mathrm{CI} 0.70-0.92, P=0.002$ ), but no significant changes for those with no mental health condition.

\section{Positive well-being}

Levels of well-being differed by age groups, with those aged 30-59 years (mean 22.01; OR $=5.78,95 \%$ CI 3.52-9.49, $P<0.0001$ ) and those aged $\geq 60$ (mean 26.01; OR $=255.59,95 \%$ CI 136.39-479.44, $P<0.0001)$ reporting higher levels of well-being than the 18 - to 29 -year-olds (mean 20.28). Men reported significantly higher well-being (mean 23.29) compared with women (mean 21.45; $\mathrm{OR}=6.17,95 \%$ CI 3.97-9.57, $P<0.0001$ ). Levels of well-being were not significantly different by ethnic group. Those of a lower SEG reported lower levels of well-being (mean 21.75; OR $=0.41$, 95\% CI 0.26-0.64, $P<0.0001)$ compared with those of a higher SEG (mean 22.66). Participants with a pre-existing mental health condition were more likely to report lower well-being scores (mean 18.64; OR $=0.007,95 \%$ CI 0.004-0.01, $P<0.0001$ ) compared with those with none (mean 23.66; see supplementary Table 8).

\section{Discussion}

\section{Main findings and comparison with findings from other studies}

This study offers a detailed examination of the mental health and well-being of the UK adult population during the first 6 weeks of 
the COVID-19 pandemic. Across every indicator, individuals from more socially disadvantaged backgrounds and those with pre-existing mental health problems report the worst mental health outcomes. The rates of suicidal ideation increased during the initial weeks of lockdown, with one in seven (14\%) young adults reporting suicidal thoughts in the last week at wave 3. It is not possible to make direct comparisons with pre-COVID-19 rates, but the rate of suicidal ideation among young adults reported here (between $12.5 \%$ and $14.4 \%$ across waves) is higher than the $11 \%$ past-year suicidal ideation rate reported by young adults in another pre-COVID-19 study. ${ }^{31}$ The weekly suicidal ideation rates for the whole sample (9.8\% at wave 3 ) are also higher than those reported elsewhere, with $2.8 \%$ reporting suicidal thoughts in one national study of adults. ${ }^{32}$

Across all three waves, approximately one in four respondents (wave $1=26.1 \%$, wave $2=24.3 \%$ and wave $3=23.7 \%$ ) experienced moderate to severe levels of depressive symptoms on the PHQ-9. This finding is concerning when compared with other preCOVID-19 general population studies where, for example, 5.6\% scored above the standard $\geq 10$ cut-off. ${ }^{33}$ However, we urge caution when extrapolating from the PHQ-9 data to general population lockdown estimates, as a recent meta-analysis concluded that the PHQ-9 may more than double the estimate of depression compared with a structured clinical interview (using the Structured Clinical Interview for Diagnostic and Statistical Manual of Mental Disorders). ${ }^{34}$ Also, although we have recruited a well-stratified national sample from across the UK, quota sampling does not guarantee the same level of representativeness as probability sampling and therefore prevalence estimates need to be interpreted accordingly. However, it is also worth noting that our depressive symptoms findings are quite similar to the latest Office for National Statistics data for the UK adult population collected in June $2020{ }^{35}$ where $19.2 \%$ of adults reported moderate to severe levels of depression, compared with $23.7 \%$ at wave 3 in our study at the end of April/start of May 2020.

For anxiety, one in five (21\%) respondents in the present sample scored above the cut-off on the GAD-7, corresponding to moderate to severe levels of anxiety at wave 1 , with this rate decreasing to $16.8 \%$ by wave 3 . We do not have pre-COVID-19 figures to make like-for-like comparisons; nonetheless, these rates were much higher than the established general population norms (of approximately 5\%). ${ }^{36}$ Levels of mental well-being among women across all waves were lower than the general population norms for the SWEMWBS, but levels for men were similar. ${ }^{26}$

\section{Implications}

As already noted, the mental health of women, of young people (1829 years), of those from more socially disadvantaged backgrounds, and of those with pre-existing mental health problems has been particularly affected during the pandemic. These groups need to be prioritised to ensure that they receive the support they require ${ }^{16}$ and accessible and remote clinical services tailored, as necessary, to meet this need. The trajectories across the three waves illustrate the importance of assessing different indicators of mental health and well-being. Whereas symptoms of anxiety, and levels of defeat and entrapment decreased across the three waves, depressive symptoms and loneliness remained stable but adversely affected. The findings also highlight that loneliness may become more of an issue for older adults as the pandemic unfolds, as well as for those from more socially disadvantaged backgrounds.

Across all of the analyses the mental health outcomes for those from ethnic minority and White backgrounds were similar. Despite our sample being well-stratified nationally, our sample size precludes a more fine-grained analysis of the mental health outcomes of people from specific ethnic minority communities. Such an analysis, that also takes account of intersectionality, is urgently required. ${ }^{1,16,17}$

The trajectories of suicidal thoughts highlight that we need to be vigilant. Although an increase in suicide is not inevitable, ${ }^{37}$ the present data may be an early indicator of emerging risk, especially as the economic fallout of COVID-19 escalates. The proportions of respondents reporting at least 1 day in the previous week that they wanted to end their life increased across the three waves of the study, from $8.2 \%$, to $9.2 \%$ and $9.8 \%$ at waves 1,2 and 3 , respectively.

Given its well established relationship with suicide risk, ${ }^{38}$ it is surprising that levels of entrapment decreased while suicidal thoughts increased. This may reflect a lagged effect or it may be that the items assessing entrapment or depression focus on the past whereas the suicidal question is tapping uncertainty or concerns about the future. This may also explain why the positive well-being measure increased, as it also focuses on the past, and likely increased as levels of anxiety decreased. The focus on future orientation is potentially crucial as future thinking is a recognised cognitive factor associated with suicidal ideation independent of depression. ${ }^{38}$ Indeed, inspection of the items to assess depressive symptoms illustrates this point as they are tapping the extent to which respondents are 'bothered' by problems in the recent past; so after the initial shock of lockdown, one's appraisal of these problems is relatively stable in the short term. By contrast, in the early weeks of the pandemic, the anticipated impact of the economic and social disruption to come may have exacerbated one's feelings of hopelessness and suicidal ideation and hence explain the increase in the latter.

Survey-based research needs to be supplemented with qualitative interviews to determine whether our conjecture about the cause of increasing levels of suicidal ideation is supported. By way of post hoc analyses, we also inspected the responses to the suicidal question in the PHQ-9 ('Thoughts that you would be better off dead, or of hurting yourself in some way?') and we find a similar pattern as above, of increasing suicidal thoughts. It is essential, therefore, that suicidal thoughts continue to be tracked as we emerge from lockdown and navigate national/local restrictions. These data are also consistent with the recent report from the National Child Mortality Database that points to a potential increase in child suicide deaths in the early stages of the pandemic. ${ }^{39}$ The defeat and entrapment levels are also of concern, especially among young adults at wave 1 . At wave 1, more than a third (37\%) of young people scored above the recommended cut-off $(>5)$ for entrapment, which indicates that further screening for suicide ideation is warranted. ${ }^{25}$

\section{Limitations}

Indicators of mental health were based on self-reports rather than clinical diagnoses, as a result, we can only comment on the trajectory of the symptoms of mental ill health rather than psychiatric disorder. Despite successfully recruiting a quota-based national sample, similar to all studies that recruit via digital means, our sample is likely to underestimate the mental health effects of COVID-19 as those who are digitally excluded may be underrepresented. Also, those who did not complete all waves tended to have worse mental health at wave 1 . Future research is required to understand what aspects of the pandemic and the pandemic response may have contributed to negative mental health outcomes as well as those factors and activities that may be protective.

To conclude, the mental health and well-being of the UK adult population appears to have been affected in the initial phase of the COVID-19 pandemic, particularly women, young adults, the socially disadvantaged and those with pre-existing mental health 
problems. The trajectory of increasing rates of suicidal thoughts, especially among young adults, is particularly concerning. These early data highlight that detailed monitoring of longer-term mental health outcomes and inequalities is essential.

Rory C. O'Connor (D), PhD, Suicidal Behaviour Research Laboratory, Institute of Health \& Wellbeing, University of Glasgow, UK; Karen Wetherall (D), PhD, Suicidal Behaviour Research Laboratory, Institute of Health \& Wellbeing, University of Glasgow, UK; Seonaid Cleare, PhD, Suicidal Behaviour Research Laboratory, Institute of Health \& Wellbeing, University of Glasgow, UK; Heather Mcclelland, MSc, Suicidal Behaviou Research Laboratory, Institute of Health \& Wellbeing, University of Glasgow, UK Ambrose J. Melson (D), PhD, Suicidal Behaviour Research Laboratory, Institute of Health \& Wellbeing, University of Glasgow, UK; ClaireL. Niedzwiedz, PhD, Institute of Health \& Wellbeing, University of Glasgow, UK; Ronan E. O'Carroll, PhD, Division of Psychology, University of Stirling, UK; Daryl B. O'Connor, PhD, School of Psychology, University of Leeds, UK; Steve Platt, PhD, Usher Institute, University of Edinburgh, UK Elizabeth Scowcroft, PhD, Samaritans, UK; Billy Watson, Scottish Association for Mental Health, UK; Tiago Zortea (D), PhD, Suicidal Behaviour Research Laboratory, Institute of Health \& Wellbeing, University of Glasgow, UK; Eamonn Ferguson, PhD, School of Psychology, Nottingham University, UK; Kathryn A. Robb, PhD, Institute of Health \& Wellbeing, University of Glasgow, UK

Correspondence: Rory C. O'Connor. Email: rory.oconnor@glasgow.ac.uk

First received 22 Jul 2020, final revision 6 Oct 2020, accepted 7 Oct 2020

\section{Supplementary material}

To view supplementary material for this article, please visit https://doi.org/10.1192/bjp.2020.212.

\section{Data availability}

The data that support the findings of this study are available from the corresponding author (R.C.O'C.), upon reasonable request.

\section{Author contributions}

R.C.O'C., K.A.R., K.W. and S.C. had the idea for the study and R.C.O'C. secured funding for the study. All authors contributed to the study design. K.W. and E.F. led the statistical analyses alongside S.C. R.C.O'C. led the drafting of the manuscript but all authors contributed to the drafting and approved the final manuscript for submission.

\section{Funding}

University of Glasgow, Samaritans, Scottish Association for Mental Health and the Mindstep Foundation.

\section{Declaration of interest}

R.C.O'C. reports grants from Samaritans, grants from Scottish Association for Mental Health grants from Mindstep Foundation, during the conduct of the study; grants from Nationa Institute for Health Research, grants from Medical Research Foundation, grants from Scottish Government, grants from NHS Health Scotland/Public Health Scotland, outside the submitted work: and he is co-chair of the Academic Advisory Group to the Scottish Government's National Suicide Prevention Leadership Group. He is also a member of the National Institute for Health and Care Excellence's guideline development group for their new self-harm guidelines. C.L.N. reports grants from Medical Research Council, during the conduct of the study. S.P. reports personal fees from Health Service Executive, Dublin, Ireland, personal fees from NHS Health Scotland, Edinburgh, Scotland, outside the submitted work. TZ reports being employed by the Scottish Government Scotland She the submitted work, reports grants from NHS Scotland Endowment Funds; and is a cochair of the Early Career Group of the International Association for Suicide Prevention. E. F. reports grants from Pfizer Limited and Versus Arthritis, grants from Versus Arthritis, grants from Football Association, grants from Medical Research Foundation, grants from U. S. Army Medical Research and Materiel Command, outside the submitted work. E.S. (Samaritans) and B.W. (Scottish Association for Mental Health) are employees of two of the funders of this research. K.W., S.C., H.MCC. A.J.M., R.E.O'C., D.B.O'C. and K.A.R. have nothing to disclose.

\section{References}

1 Holmes EA, O'Connor RC, Perry VH, Tracey I, Wessely S, Arseneault L, et al. Multidisciplinary research priorities for the COVID-19 pandemic: a call for action for mental health science. Lancet Psychiatry 2020; 7: 547-60.
2 Hotopf M, Bullmore E, O'Connor RC, Holmes EA. The scope of mental health research during the COVID-19 pandemic and its aftermath. Br J Psychiatry 2020; 217: 540-542.

3 Cheung YT, Chau PH, Yip PSF. A revisit on older adults suicides and severe acute respiratory syndrome (SARS) epidemic in Hong Kong. Int J Geriatr Psychiatry 2008; 23: 1231-8.

4 Yip PSF, Cheung YT, Chau PH, Law YW. The impact of epidemic outbreak the case of Severe Acute Respiratory Syndrome (SARS) and suicide among older adults in Hong Kong. Crisis 2010; 31: 86-92.

5 Lee SM, Kang WS, Cho A-R, Kim T, Park JK. Psychological impact of the 2015 MERS outbreak on hospital workers and quarantined hemodialysis patients. Compre Psychiatry 2018; 87: 123-7.

6 Rogers JP, Chesney E, Oliver D, Pollak TA, McGuire P, Fusar-Poli P, et al. Psychiatric and neuropsychiatric presentations associated with severe coronavirus infections: a systematic review and meta-analysis with comparison to the COVID-19 pandemic. Lancet Psychiatry 2020; 7: 611-27.

7 Mak IWC, Chu CM, Pan PC, Yiu MGC, Chan VL. Long-term psychiatric morbidities among SARS survivors. Gen Hosp Psychiatry 2009; 31: 318-26.

8 Gao J, Zheng P, Jia Y, Chen H, Mao Y, Chen S, et al. Mental health problems and social media exposure during COVID-19 outbreak. PLoS One 2020; 15: e0231924-e.

9 Tian F, Li H, Tian S, Yang J, Shao J, Tian C. Psychological symptoms of ordinary Chinese citizens based on SCL-90 during the level I emergency response to COVID-19. Psychiatry Res [Epub ahead of print] 11 Apr 2020. Available from: https://doi.org/10.1016/j.psychres.2020.112992.

10 Wang C, Pan R, Wan X, Tan Y, Xu L, Ho CS, et al. Immediate psychological responses and associated factors during the initial stage of the 2019 coronavirus disease (COVID-19) epidemic among the general population in China. I. Int $J$ Environ Res Public Health 2020; 17: 1729.

11 Wang C, Pan R, Wan X, Tan Y, Xu L, McIntyre RS, et al. A longitudinal study on the mental health of general population during the COVID-19 epidemic in China. Brain Behav Immun [Epub ahead of print] 13 Apr 2020. Available from: https://doi.org/10.1016/j.bbi.2020.04.028.

12 Losada-Baltar A, Jiménez-Gonzalo L, Gallego-Alberto L, Pedroso-Chaparro MDS, Fernandes-Pires J, Márquez-González M. We're staying at home. Association of self-perceptions of aging, personal and family resources and loneliness with psychological distress during the lockdown period of COVID-19. J Gerontol B Psychol Sci Soc Sci [Epub ahead of print] 13 Apr 2020. Available from: https://doi.org/10.1093/geronb/gbaa048.

13 Lob E, Steptoe A, Fancourt D. Abuse, self-harm and suicidal ideation in the UK during the COVID-19 pandemic. Br J Psychiatry 2020; 217: 543-6.

14 Pierce $\mathrm{M}$, Hope $\mathrm{H}$, Ford $\mathrm{T}$, Hatch $\mathrm{S}$, Hotopf $\mathrm{M}$, John $\mathrm{A}$, et al. Mental health before and during the COVID-19 pandemic: a longitdinal probability sample survey of the UK population. Lancet Psychiatry 2020; 7: 883-92.

15 Douglas M, Katikireddi SV, Taulbut M, McKee M, McCartney G. Mitigating the wider health effects of Covid-19 pandemic response. BMJ [Epub ahead of print] 27 Apr 2020. Available from: https://doi.org/10.1136/bmj.m1557.

16 O'Connor RC, Hotopf M, Worthman CM, Perry VH, Tracey I, Wessley S, et al. Multidisciplinary research priorities for the COVID-19 pandemic: authors' reply. Lancet Psychiatry 2020; 7: e44-45

17 O'Connor DB, Aggleton JP, Chakarabarti B, Armitage CJ. Research priorities for the COVID-19 pandemic and beyond: a call to action for psychological science. Br J Psychol [Epub ahead of print] 19 Jul 2020. Available from: https://doi.org/ 10.1111/bjop. 12468

18 National Readership Survey. Social Grade. National Readership Survey 2016 (http://www.nrs.co.uk/nrs-print/lifestyle-and-classification-data/socialgrade/).

19 UK Government. Ethnicity Facts and Figures. gov.uk, 2018 (https://www.ethnicity-facts-figures.service.gov.uk/uk-population-by-ethnicity/demographics/ age-groups/latest-main-facts-and-figures).

20 McManus S, Hassiotis A, Jenkins R, Dennis M, Aznar C, Appleby L. Chapter 12 : Suicidal thoughts, suicide attempts and self-harm. In Mental Health and Wellbeing in England: Adult Psychiatric Morbidity Survey 2014 (eds S McManus, P Bebbington, R Jenkins, T Brugha): 294-322. NHS Digital, 2016.

21 Löwe B, Kroenke K, Herzog W, Gräfe K. Measuring depression outcome with a brief self-report instrument: sensitivity to change of the Patient Health Questionnaire (PHQ-9). J Affect Disord 2004; 81: 61-6.

22 Spitzer RL, Kroenke K, Williams JB, Lowe B. A brief measure for assessing generalized anxiety disorder: the GAD-7. Arch Intern Med 2006; 166 1092-7.

23 Kroenke K, Spitzer RL. The PHQ-9: a new depression diagnostic ans severity measure. Psychiatr Ann 2002; 1: 509-15.

24 Griffiths AW, Wood AM, Maltby J, Taylor PJ, Panagioti M, Tai S. The development of the Short Defeat and Entrapment Scale (SDES). Psychol Assess 2015; 27: 1182-94. 
25 De Beurs D, Cleare S, Wetherall K, Eschle-Byrne S, Ferguson E, O'Connor DB et al. Entrapment and suicide risk: the development of the 4-item Entrapment Scale Short-Form (E-SF). Psychiatry Res [Epub ahead of print] 9 Jan 2020. Available from: https://doi.org/10.1016/j.psychres.2020.112765.

26 Fat LN, Scholes S, Boniface S, Mindell J, Stewart-Brown S. Evaluating and establishing national norms for mental wellbeing using the short WarwickEdinburgh Mental Well-being Scale (SWEMWBS): findings from the Health Survey for England. Qual Life Res 2017; 26: 1129-44.

27 Hughes ME, Waite LJ, Hawkley LC, Cacioppo JT. A short scale for measuring loneliness in large surveys - results from two population-based studies. Res Aging 2004; 26: 655-72.

28 Beunckens C, Sotto C, Molenberghs G. A simulation study comparing weighted estimating equations with multiple imputation based estimating equations for Iongitudinal binary data. Comput Stat Data Anal 2008; 52: 1533-48.

29 Ballinger GA. Using generalized estimating equations for longitudinal data analysis. Organ Res Methods 2004; 7: 127-50.

30 Benjamini $Y$, Hochberg Y. Controlling the false discovery rate: a practical and powerful approach to multiple testing. J R Stat Soc Ser A 1995; 57: 289-300.

31 O'Connor RC, Wetherall K, Cleare S, Eschle S, Drummond J, Ferguson E, et al. Suicide attempts and non-suicidal self-harm: national prevalence study of young adults. Br J Psychiatry Open 2018; 4: 142-8.

32 Lee J-I, Lee M-B, Liao S-C, Chang C-M, Sung S-C, Chiang H-C, et al. Prevalence of suicidal ideation and associated risk factors in the general population. J Formosan Med Assoc 2010; 109: 138-47.
33 Kocalevent R-D, Hinz A, Braehler E. Standardization of the depression screener Patient Health Questionnaire (PHQ-9) in the general population. Gen Hosp Psychiatry 2013; 35: 551-5.

34 Levis B, Benedetti A, Ioannidis JPA, Sun Y, Negeri Z, He C, et al. Patient Health Questionnaire-9 scores do not accurately estimate depression prevalence: individual participant data meta-analysis. J Clin Epidemiol 2020; 122: $115-28$.

35 Office for National Statistics. Coronavirus and Depression in Adults. ONS, 2020

36 Lowe B, Decker O, Müller S, Brähler E, Schellberg D, Herzog W, et al. Validation and standardization of the Generalized Anxiety Disorder Screener (GAD-7) in the general population. Med Care 2008; 46: 266-74.

37 Gunnell D, Appleby L, Arensman E, Hawton K, John A, Kapur N et al. Suicide risk and prevention during the COVID-19 pandemic. Lancet Psychiatry 2020; 7: 468-71.

38 O'Connor RC, Kirtley OJ. The integrated motivational-volitional model of suicidal behaviour. Philos Trans R SOCB 2018; 373: 20170268.

39 Odd D, Sleap V, Appleby L, Gunnell D, Luyt K. Child suicide Rates during the COVID-19 Pandemic in England: Real-Time Surveillance. National Child Mortality Database, 2020.

\section{psychiatry in history}

\section{Epidemics: the first pandemic - the Justinianic Plague (541-549)}

\section{Greg Wilkinson (D)}

The Early Medieval Pandemic began in 541 with the Justinianic Plague (Yersinia pestis) and continued through the 8th century. Today, echoes sound from the accounts of Procopius (c. 500 to after 565), Evagrius Scholasticus (born c. 536) and John of Ephesus (c. 507-588).

The historian Procopius says that pestilence embraced the entire world and blighted the lives of all men, respecting neither gender nor age, attacking at all times of the year, starting in Egypt, then to Palestine and on to the land of the Persians and barbarians (Dewing 1914). There was sudden fever, and within days a bubonic swelling developed in the groin or elsewhere. Some became comatose, forgot those familiar to them and seemed to be sleeping. If anyone cared for them, they would eat without waking; but some were neglected, and they died through lack of sustenance. Others developed delirium, with insomnia and distorted imagination: they suspected men were coming to destroy them, and would rush off in flight, crying out. Those attending them were in a state of constant exhaustion and had a most difficult time of it. Work of every description ceased, and in a city abounding in good things starvation ran riot.

Evagrius, scholar and intellectual, describes being seized with buboes while still a schoolboy, and losing by recurrence of the plague at different times his wife, several children, many kin, as well as domestic and country servants; not quite 2 years before writing, now in his 58th year, on its fourth visit to Antioch, he lost a daughter and her son (Walford 1846). Manifestations were various. Some died after 2 or 3 days, in possession of their mental and bodily powers. People who had been attacked once and twice and had recovered, died by a subsequent occurrence. Some perished by living with the infected; by touching them; by having entered their chamber; or by frequenting public places. Some fled infected cities but imparted the disease to the healthy. Some were free from contagion, although they had associated with many afflicted in sickness and death. Some who desired death, on account of the loss of their children and friends, placed themselves as much as possible in contact with the diseased, but nevertheless were not infected.

John of Ephesus was a Byzantine historian. Much of his Ecclesiastical History is missing but part appears in the Chronicle of patriarch Michael the Syrian (1126-1199) (Chabot 1899). John was living in Constantinople when the scourge fell: 'men became enraged, like dogs, went mad, attacked each other, went into the mountains and committed suicide'. It raged among the poor; there were days when 5000 were carried away, others 7000, others 12 000, and up to 16000 in one day; over 300000 were taken from public places. When those of low rank were dead, the devastator stretched out his hand over the mighty; not forgetting animals and reptiles.

(c) The Author(s), 2021. Published by Cambridge University Press on behalf of the Royal College of Psychiatrists 